\title{
Brain sexual differentiation: Clues toward the understanding of neural dysfunctions
}

\author{
Jessica A. Mong • Margaret M. McCarthy
}

Published online: 14 July 2012

(C) Springer Science+Business Media, LLC 2012

Despite the ever-growing recognition of sex disparities in the prevalence of and susceptibility to neural dysfunctions, the need for understanding the basic etiology of sex differences in the brain remains undervalued. A point evidenced by the exclusion of females in many basic research and clinical studies. Nevertheless, sex differences in brain morphology, physiology, and behaviors have been well studied. Additionally, epidemiological analysis of central nervous system disorders reveals a salient pattern of sex differences in the human population. Neuropsychiatric and learning disorders with developmental origins such as autism spectrum disorders, attention deficit disorders and dyslexia are more prevalent in males whereas the incidence of mental health dysfunctions (e.g., schizophrenia, anorexia/bulimia and affective disorders) and aging-related neurodegenerative diseases (e.g., stroke and Alzheimer's Disease) are more prevalent during transitional periods in the lifespan of girls and women that coincide with changes in the ovarian hormone milieu.

More than a half-century of work clearly demonstrates a prominent role of sex steroids (estrogens, androgens and progestins) in the developmental organization of the mammalian brain (including humans) into a masculinized or feminized phenotype. In what is now considered a 'classical view' of this developmental process, the fetal testes release a surge of androgens during a restricted developmental window that acts throughout the body to masculinize steroid

\footnotetext{
J. A. Mong $(\bowtie) \cdot$ M. M. McCarthy

Department of Pharmacology,

University of Maryland, School of Medicine,

655 West Baltimore St,

Baltimore, MD 21201, USA

e-mail: jmong001@umaryland.edu

M. M. McCarthy

e-mail: mmccarth@umaryland.edu
}

responsive tissues including the brain. In females, in the absence of an androgen surge, feminized neural patterns develop; a developmental program that has been traditionally viewed as a "default" pathway. These processes of masculinization and feminization permanently change or organize the neural environment of the organism in a sexspecific manner so that later in adult life sex-specific gonadal steroids will transiently activate the neural patterns leading to sex differences in CNS function.

In recent years the organizational/activational dogma of brain sex differentiation has met some challenges that are reshaping the way we think about how sex differences in the brain and ultimately behavior are established and how understanding these differences may gives important insights into the origins and maintenance of CNS dysfunctions. The reviews in this special issue represent emergent new directions in mechanisms underlying brain sexual differentiation as well as the role of sex steroids in mental and neurological pathologies. Starting with sex chromosome compliment, Drs. Abel and Rissman discuss the genetic bases for several well-described sex differences in protein expression that were traditionally thought to be mediated purely by the organizational actions of gonadal steroids. Drs. Matsuda, Mori and Kawata present a convincing case for the role of epigenetic modifications of steroid receptors in brain masculinization. Dr. Nuñez discusses the intersection of genetic sex and developmental exposure to sex steroids on outcomes in early brain injury. Lastly, Dr. Markham discusses the rise in sex steroids during puberty as a time of increased susceptibility to psychiatric illness.

Today, scientists and clinicians, must consider a complex set of interactions between steroids, sex chromosomes and environmental factors when thinking about CNS-related sex differences. In summary, this special issue reviews our new understandings of how sex differences in the brain are established. 